Transactions on Alogorithms 1 (2005), 123-142

\title{
ANALYSIS OF LINEAR COMBINATION ALGORITHMS IN CRYPTOGRAPHY
}

\author{
PETER J. GRABNER ${ }^{\dagger}$, CLEMENS HEUBERGER ${ }^{\ddagger}$, HELMUT PRODINGER* $^{*}$ \\ AND JÖRG M. THUSWALDNER ${ }^{\top}$
}

\begin{abstract}
Several cryptosystems rely on fast calculations of linear combinations in groups. One way to achieve this is to use joint signed binary digit expansions of small "weight." We study two algorithms, one based on non adjacent forms of the coefficients of the linear combination, the other based on a certain joint sparse form specifically adapted to this problem. Both methods are sped up using the sliding windows approach combined with precomputed lookup tables. We give explicit and asymptotic results for the number of group operations needed assuming uniform distribution of the coefficients. Expected values, variances and a central limit theorem are proved using generating functions.

Furthermore, we provide a new algorithm which calculates the digits of an optimal expansion of pairs of integers from left to right. This avoids storing the whole expansion, which is needed with the previously known right to left methods, and allows an online computation.
\end{abstract}

\section{INTRODUCTION}

In many public key cryptosystems, raising one or more elements of a given group to large powers plays an important role (cf. for instance $[2,9]$ ). In practice, the underlying groups are often chosen to be the multiplicative group of a finite field $\mathbb{F}_{q}$ or the group law of an elliptic curve (elliptic curve cryptosystems).

Let $P$ be an element of a given group, whose group law is written additively throughout the paper. What we need is to form $n P$ for large $n \in \mathbb{N}$ in a short amount of time. One way to do this is the binary method (cf. [14]). This method uses the operations of "doubling" and "adding $P$ ". If we write $n$ in its binary representation, the number of doublings is fixed by $\left\lfloor\log _{2} n\right\rfloor$ and each one in this representation corresponds to an addition. Thus the cost of the multiplication depends on the length of the binary representation of $n$ and the number of ones in this representation. The goal of the methods presented in this paper is to decrease the cost by finding representations of integers containing few nonzero digits.

If addition and subtraction are equally costly in the underlying group, it makes sense to work with signed binary representations, i.e., binary representations with digits $\{0, \pm 1\}$. The advantage of these representations is their redundancy: in general, $n$ has many different signed binary representations. Let $n$ be written in a signed binary representation. Then the number of non-zero digits is called the Hamming weight of this representation. Since each non-zero digit causes a group addition ( 1 causes addition of $P,-1$ causes subtraction of $P$ ), one is interested in finding a representation of $n$ having minimal Hamming weight. Such a minimal representation was exhibited by Reitwiesner [11]. Since it has no adjacent non-zero digits, this type of representation is often called non-adjacent form or NAF, for short. On average, only one third of the digits of a NAF is different from zero. Morain and Olivos [10] first observed that NAFs are useful for calculating $n P$ for large $n$ quickly.

Date: February 14, 2008.

2000 Mathematics Subject Classification. Primary: 11A63; Secondary: 94A60, 68W40.

Key words and phrases. Hamming weight, digital expansions, online algorithm, elliptic curve cryptosystems.

$\dagger$ This author is supported by the START-project Y96-MAT of the Austrian Science Fund.

$\ddagger$ This author is supported by the grant S8307-MAT of the Austrian Science Fund.

* This author is supported by the grant NRF 2053748 of the South African National Research Foundation.

I This author is supported by the grant S8310-MAT of the Austrian Science Fund. 
Recently, Solinas [12] considered the problem of computing $m P+n Q$ at once, without computing each of the summands separately. Using unsigned binary representations of $m$ and $n$ this can be done with the help of the operations "doubling" and "adding $P, Q$, or $P+Q$ ". We are again interested in diminishing the number of additions. Assume that the additions of the three quantities are equally costly. If we write the binary representations $m=\sum a_{j} 2^{j}$ and $n=\sum b_{j} 2^{j}$ in the form ${ }_{b_{\ell}}^{a_{\ell}} \ldots{ }_{b_{0}}^{a_{0}}$, the cost of the calculation of $m P+n Q$ depends on the number of nonzero columns in this joint representation. This number is called the joint Hamming weight of this (joint) representation. If addition and subtraction are equally costly, again by using signed representations of $m$ and $n$, one can reduce the joint Hamming weight considerably (note that for signed representations we have to deal with the addition of $\pm P, \pm Q, \pm P \pm Q$ and $\pm P \mp Q$ ). One way to do this consists in writing $m$ and $n$ in their NAF. However, in the above mentioned paper, Solinas found an even "cheaper" way of representing $m$ and $n$ : the so called Joint Sparse Form. It turns out that his construction yields the minimal joint Hamming weight among all joint expansions of two numbers. In Grabner et al. [3] this concept was simplified and extended to the joint representation of $d \geq 2$ numbers and its properties are studied in detail. The representation used in [3] is therefore called Simple Joint Sparse Form, or SJSF for short.

The detailed definitions of joint NAFs and SJSF for $d$ integers are given in Section 2 and Section 3, respectively.

In all these algorithms we determined $n P$ and $m P+n Q$ by doubling and adding some quantities. There is a modification of these algorithms by using so called windows or window methods (cf. for instance Gordon [2, Section 3] or Avanzi [1]). This is a rather easy concept. We explain it for the case of the computation of $m P+n Q$ with $m, n$ written in binary representation. First select a window size $w$. Then precompute all sums of the form $r P+s Q$ such that $r$ and $s$ have a binary representation of length at most $w$. Now we can compute $m P+n Q$ by multiplying by $2^{w}$ and adding one of the precomputed values. Of course, this makes the algorithms faster at the cost of precomputation tables. There are many ways to refine this concept and to consider adaptations which are suitable to special representations. An easy modification consists in jumping over zero vectors at the beginning of a window. If we use window methods where zero digits are forced after a bounded number of non-zero digits we may adapt the size of the window after each step in order to exploit these zeros. The latter modification is possible for instance in the case of SJSF. We explain all this in more detail when we apply windows to our algorithms later.

In the present paper we are concerned with the joint representation of $d$-tuples of integers. In Section 2 we dwell upon joint NAFs with windows. In particular, we give a detailed analysis of the average cost of calculating linear combinations $n_{1} P_{1}+\cdots+n_{d} P_{d}$ by examining the joint Hamming weight of joint NAFs. We give expressions of the average cost, its variance as well as its distribution. This extends and refines the work of Avanzi [1].

In Section 3 we perform a detailed runtime analysis of the SJSF of $d$ integers using window methods. Contrary to the joint NAF the SJSF guarantees that after at most $d$ non-zero columns (or digits) there occurs a zero column. It is natural to adapt the size of the windows dynamically in a way that we can expect zero columns at the beginning of each new window. In this way we can exploit the existing zeros in an optimal way. In this case it is a nontrivial problem to compute the size of the precomputation tables. We give an asymptotic formula for their size. We note that Solinas [12] does not consider windows and Avanzi [1] considers Solinas' Joint Sparse Form with a fixed window size of 2 .

From the way we calculate the linear combinations $n_{1} P_{1}+\cdots+n_{d} P_{d}$ we see that we proceed through the representations of $n_{1}, \ldots, n_{d}$ starting from their most significant digit down their least significant digit, or, in other words, from left to right. Unfortunately, as Avanzi [1] and Solinas [12] both regret, the known algorithms for the SJSF produce the representations from right to left. This has the disadvantage that we need to calculate the whole SJSF representation from right to left before we can start to apply it from left to right in order to compute our linear combinations. Especially if we have to deal with long representations this requires a large amount of memory.

Our last section, however, is devoted to a remedy to this unfortunate situation by providing a transducer (with 32 "essential" states) which constructs a minimal joint representation from 
left to right for $d=2$. This is done by first writing each of the numbers $m, n$ separately in a representation which gives us some freedom in changing their digits locally reading from the left. Because of its resemblance to the well-known greedy expansion we call this representation the alternating greedy expansion. Starting from this expansion we succeeded in constructing a minimal joint representation of $m, n$ from left to right. In contrast to [3] which gives a fractal description of the SJSF from left to right, this new minimal representation is computable from left to right.

\section{JOINT NON-ADJACENT FORM}

The present section is devoted to the complexity analysis of the joint distribution of integers in non-adjacent form. Recall that a NAF is a signed binary representation of an integer $x$ of the shape

$$
x=\sum_{j=0}^{J} x_{j} 2^{j} \quad \text { with } \quad x_{j} \in\{0, \pm 1\}
$$

such that $x_{j} x_{j+1}=0$ for all $j \in\{0, \ldots, J-1\}$. For a given integer it is possible to compute its NAF with help of the easy Algorithm 1.

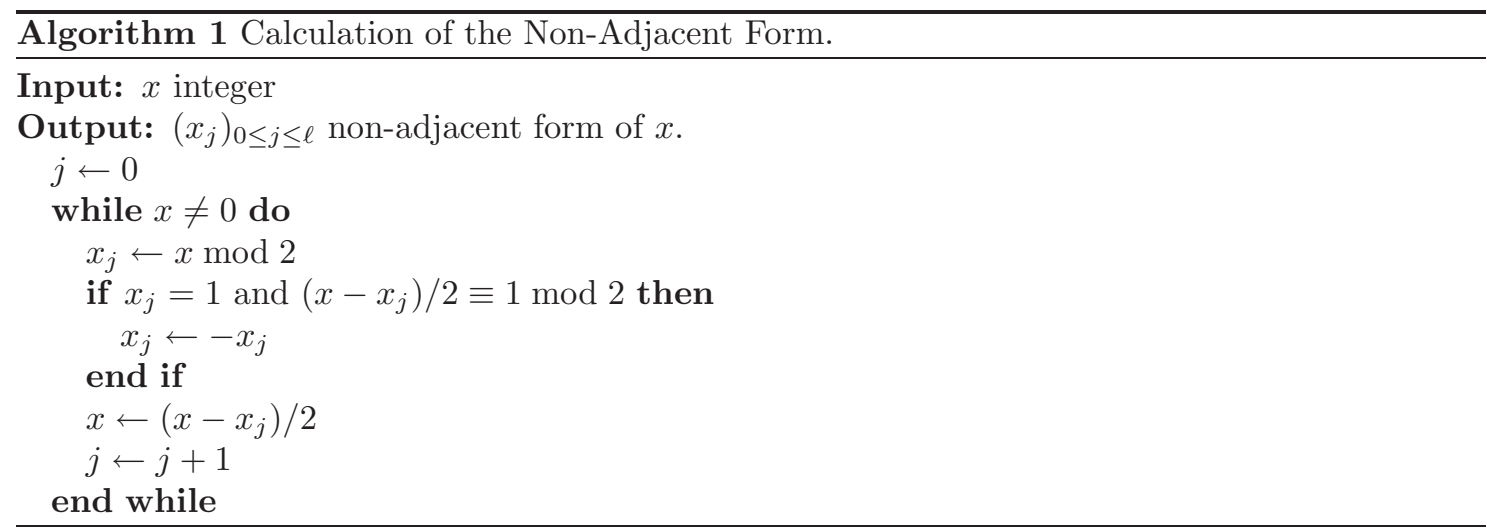

Note that Algorithm 1 is the same as the algorithm for computing the simple joint sparse form for $d=1$ (see Section 3). This algorithm can easily be interpreted as a three state transducer (cf. Figure 1).

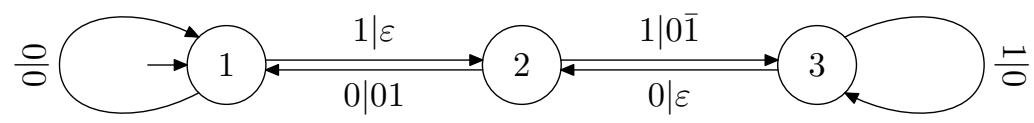

Figure 1. Transducer to compute the NAF from right to left.

Using this transducer an easy calculation yields that the expected value of the Hamming weight of an expansion of length $J$ is asymptotically $J / 3$ (cf. [13]).

Let $d \in \mathbb{N}$. In what follows we investigate $d$-tuples of NAFs. Such a $d$-tuple is called joint $N A F$. Joint NAFs can be regarded as finite sequences of $d$-dimensional vectors. We write $d$-dimensional vectors in boldface. For the coordinates of a vector we use the notational convention

$$
\mathbf{x}=\left(x^{(1)}, \ldots, x^{(d)}\right) .
$$

We set up an easy probabilistic model. Define the space

$$
\mathcal{N}_{d}:=\left\{\left(\ldots, \mathbf{x}_{1}, \mathbf{x}_{0}\right) \in\{0, \pm 1\}^{d \times \mathbb{N}_{0}} \mid \forall j \in \mathbb{N}_{0}, k \in\{1, \ldots, d\}: x_{j}^{(k)} x_{j+1}^{(k)}=0\right\}
$$

whose elements are called infinite joint NAFs. On $\mathcal{N}_{d}$ we define a probability measure by the image of the Haar measure on $\mathbb{Z}_{2}^{d}:=\{0,1\}^{d \times \mathbb{N}_{0}}$ via the map $\mathbb{Z}_{2}^{d} \rightarrow \mathcal{N}_{d}$ given by Algorithm 1 . The joint Hamming weight of a joint $\operatorname{NAF}\left(\mathbf{x}_{j}\right)_{j \geq 0}$ is the number of $j \in \mathbb{N}_{0}$ with $\mathbf{x}_{j} \neq \mathbf{0}$. In order to 
derive results on the distribution of the Hamming weight of NAFs we need information on the number of nonzero entries in a vector $\mathbf{x}_{j}$. Thus we set

$$
A(\mathbf{x}):=\left\{k \in\{1, \ldots, d\} \mid x^{(k)} \neq 0\right\} .
$$

Let $\mathbf{x}, \mathbf{y} \in\{0, \pm 1\}^{d}$ satisfying $x^{(k)} y^{(k)}=0$ for all $k \in\{1, \ldots, d\}$. We define the random variable $\mathbf{X}_{j}$ to be the $j$-th column of an infinite joint NAF. Then, keeping track of Algorithm 1 for each of the coordinates, we derive

$$
\mathbb{P}\left(\mathbf{X}_{j+1}=\mathbf{y} \mid \mathbf{X}_{j}=\mathbf{x}\right)=2^{-d-\# A(\mathbf{y})+\# A(\mathbf{x})}
$$

and

$$
\mathbb{P}\left(\mathbf{X}_{0}=\mathbf{y}\right)=2^{-d-\# A(\mathbf{y})} .
$$

As mentioned above, we are only interested in the Hamming weight of joint NAFs. Thus it suffices to consider the random variables $\# A\left(\mathbf{X}_{j}\right)$ rather than $\mathbf{X}_{j}$ itself. Using (2.2) we easily derive

$$
p_{k, \ell}:=\mathbb{P}\left(\# A\left(\mathbf{X}_{j+1}\right)=\ell \mid \# A\left(\mathbf{X}_{j}\right)=k\right)=2^{-d+k}\left(\begin{array}{c}
d-k \\
\ell
\end{array}\right) .
$$

These quantities will be helpful in order to study the number of group additions required for multiple exponentiation algorithms which are used in cryptography (cf. Avanzi [1]). As mentioned in the introduction, such algorithms can be accelerated by using window methods (cf. for instance Gordon [2]). Suppose we want to compute the linear combination $x^{(1)} P_{1}+\cdots+x^{(d)} P_{d}$ in an Abelian group $G$ using joint NAFs with window length $w$. Then we need a table of precomputed values given by

$$
\begin{aligned}
\operatorname{PreComp}_{d, w}: & = \\
& \left\{\sum_{j=0}^{w-1} 2^{j}\left(y_{j}^{(1)} P_{1}+\cdots+y_{j}^{(d)} P_{d}\right) \mid\left(\mathbf{y}_{0}, \ldots, \mathbf{y}_{w-1}\right) \in\{0, \pm 1\}^{d \times w}, \mathbf{y}_{0} \neq 0\right\} /\{ \pm 1\} .
\end{aligned}
$$

It is clear that larger windows lead to less group additions at the cost of larger precomputation tables on the other hand. From Avanzi [1] we know that

$$
\# \operatorname{PreComp}_{d, w}=\frac{I_{w}^{d}-I_{w-1}^{d}}{2} \quad \text { with } \quad I_{w}:=\frac{2^{w+2}-(-1)^{w}}{3} .
$$

This follows easily by noting that $I_{w}$ is equal to the number of NAFs of length $w$, which can be computed by analyzing Algorithm 1.

We now want to examine Algorithm 2 described by Avanzi [1], which produces joint NAFs using windows. In particular, we want to derive distribution results for the random variable $W_{n, w}$ which counts the number of group additions in $G$ when this algorithm is applied to $\left(\mathbf{X}_{n-1}, \ldots, \mathbf{X}_{0}\right)$ (i.e., $W_{n, w}$ counts the number of windows that are "opened" by Algorithm 2, in other words, it counts how many group additions are required in order to compute a linear combination of group elements using the joint NAF). Since $w$ is fixed we write $W_{n}$ instead of $W_{n, w}$.

To this matter we study the bivariate generating function

$$
F(y, z):=\sum_{m=0}^{\infty} \sum_{n=0}^{\infty} \mathbb{P}\left(W_{n}=m\right) y^{m} z^{n} .
$$

In order to get a closed expression for this function we note first that in view of Algorithm 2 each $d$-tuple of NAFs can be written using the regular expression

$$
\left(0^{*} N B^{w-1}\right)^{*} 0^{*}\left\{\varepsilon, N, N B, \ldots, N B^{w-2}\right\} .
$$

Here $B:=\{0, \pm 1\}^{d}, N:=B \backslash\{\mathbf{0}\}$, and $\varepsilon$ is the empty word. In addition, each coordinate has to satisfy the NAF condition. Note that each occurrence of $N$ in this regular expression causes a group addition in Algorithm 2. Thus, we have to label each occurrence of $N$ with $y$. Labelling 


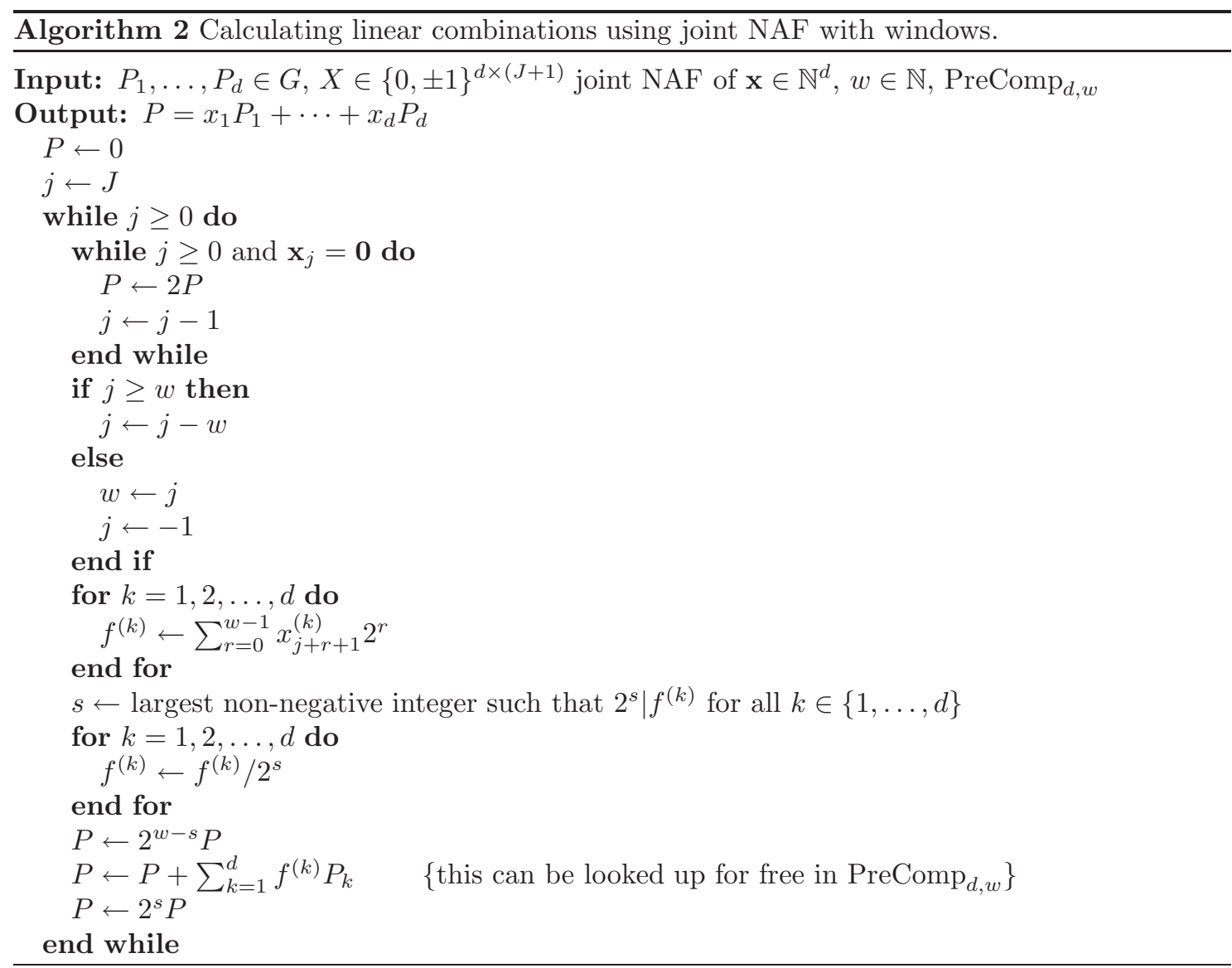

each digit with $z$ leads to the desired function. As usual, we encode the Markov chain defined by $p_{k, \ell}$ by matrices. Denote the $(d+1)$-dimensional identity matrix by $I$ and set

$$
\begin{aligned}
P & :=z\left(p_{\ell, k}\right)_{0 \leq k, \ell \leq d}, \\
Z & :=z\left([k=0] p_{\ell, k}\right)_{0 \leq k, \ell \leq d}, \\
G & :=z\left([k>0] p_{\ell, k}\right)_{0 \leq k, \ell \leq d},
\end{aligned}
$$

where Iverson's notation, popularized in [5], has been used: $[P]$ is defined to be 1 if condition $P$ is true, and 0 otherwise. Translating (2.4) into generating functions we get

$$
F(y, z)=(1, \ldots, 1) S(y, z) T(y, z)(1,0, \ldots, 0)^{T}
$$

with

$$
\begin{aligned}
& S(y, z):=\left(I-(I-Z)^{-1} y G P^{w-1}\right)^{-1}, \\
& T(y, z):=(I-Z)^{-1}\left(I+y G\left(I-P^{w-1}\right)(I-P)^{-1}\right) .
\end{aligned}
$$

We mention that $S$ represents the expression $\left(0^{*} N B^{w-1}\right)^{*}$ in $(2.4)$, while $T$ represents the possible tails after this expression. The vectors at the left and right hand side of the matrix expression for $F$ can be explained as follows. Because of

$$
\mathbb{P}\left(\mathbf{X}_{1}=\mathbf{y}\right)=\mathbb{P}\left(\mathbf{X}_{1}=\mathbf{y} \mid \mathbf{X}_{0}=\mathbf{0}\right)
$$

we always start with the digit vector $\mathbf{0}$. On the other hand, we can end up with an arbitrary digit vector.

One can easily imagine that the expression (2.5) becomes more and more complex for increasing dimensions $d$. Using Mathematica ${ }^{\circledR}$, we computed $F$ explicitly for $1 \leq d \leq 5$. To give an 
impression of the expressions obtained, we include the resulting generating function for $d=1$ :

$$
F(y, z)=\frac{4 y(1-z) z^{w}-(-2)^{w}\left(6-2 y z^{w}-z\left(6-y\left(3-z^{w}\right)\right)\right)}{(1-z)\left(4 y(1-z) z^{w}-(-2)^{w}\left(6-2 y z^{w}-z\left(3+y z^{w}\right)\right)\right)} .
$$

Since these expressions become very large for $d \geq 2$, we refrain from writing them down here and refer to the file which is available at [4].

As usual, the generating functions of $\mathbb{E}\left(W_{n}\right)$ and $\mathbb{E}\left(W_{n}\left(W_{n}-1\right)\right)$ are computed by taking the first and second derivative w.r.t. $y$, respectively, and setting $y=1$. From this we can easily calculate the variance $\mathbb{V}\left(W_{n}\right)$. In view of $(2.5)$ it is clear that for each choice of $(d, w)$ we obtain a rational function $F$. The main term in the asymptotic expansion of $\mathbb{E}\left(W_{n}\right)$ and $\mathbb{V}\left(W_{n}\right)$ comes from the dominant double and triple pole of $F$, respectively. We state exactly those results which fit into one line, the others (main terms and constant terms for $\mathbb{E}\left(W_{n}\right)$ and $\mathbb{V}\left(W_{n}\right)$ and $1 \leq d \leq 5$ ) are available at [4]. For $d=1$, we have

$$
\begin{aligned}
& \mathbb{E}\left(W_{n}\right)=\frac{3(-2)^{w}}{(-2)^{w}(4+3 w)-4} n+\frac{3(-2)^{w} w\left(-8-(-2)^{w}+3(-2)^{w} w\right)}{2\left(-4+4(-2)^{w}+3(-2)^{w} w\right)^{2}}+O\left(\rho_{w}^{n}\right), \\
& \mathbb{V}\left(W_{n}\right)=\frac{12\left(5(-8)^{w}-4^{w}-4(-2)^{w}\right)}{\left((-2)^{w}(4+3 w)-4\right)^{3}} n+O_{w}(1)
\end{aligned}
$$

for some $\left|\rho_{w}\right|<1$. For $d=2$, we get

$$
\begin{aligned}
& \mathbb{E}\left(W_{n}\right)=\frac{3(-2)^{w}\left(8+9(-2)^{w}\right)}{-16+16 \cdot 4^{w}+24(-2)^{w} w+27 \cdot 4^{w} w} n+O_{w}(1), \\
& \mathbb{V}\left(W_{n}\right)=\frac{48\left(-1+(-2)^{w}\right)(-2)^{w}\left(1+(-2)^{w}\right)\left(128+464(-2)^{w}+560 \cdot 4^{w}+261(-8)^{w}\right)}{\left(-16+16 \cdot 4^{w}+24(-2)^{w} w+27 \cdot 4^{w} w\right)^{3}} n+O_{w}(1),
\end{aligned}
$$

and for $d=3$,

$$
\begin{aligned}
& \mathbb{E}\left(W_{n}\right) \sim \\
& \frac{9(-2)^{w}\left(16+36(-2)^{w}+37 \cdot 4^{w}+21(-8)^{w}\right)}{-64-64(-2)^{w}+64(-8)^{w}+64 \cdot 16^{w}+144(-2)^{w} w+324 \cdot 4^{w} w+333(-8)^{w} w+189 \cdot 16^{w} w} n .
\end{aligned}
$$

We list these main terms for the pairs $(d, w)$ with $1 \leq d \leq 5$ and $w \leq 3$ in Table 1 .

Since the generating function $F(y, z)$ fits into the general scheme of H.-K. Hwang's "quasi-power theorem" (cf. [7]), the random variable $W_{n}$ satisfies a central limit theorem

$$
\lim _{n \rightarrow \infty} \mathbb{P}\left(W_{n} \leq \mathbb{E}\left(W_{n}\right)+x \sqrt{\mathbb{V}\left(W_{n}\right)}\right)=\frac{1}{\sqrt{2 \pi}} \int_{-\infty}^{x} e^{-\frac{t^{2}}{2}} d t .
$$

Theorem 1. Let $w \geq 1, d \geq 1$ and $W_{n, w}$ be the random variable counting the number of group additions when calculating $X_{1} P_{1}+\cdots+X_{d} P_{d}$ using Algorithm 2, where $X_{1}, \ldots, X_{d}$ are independent random variables uniformly distributed on $\left\{0, \ldots, 2^{n}-1\right\}$. Then

$$
\lim _{n \rightarrow \infty} \mathbb{P}\left(W_{n, w} \leq \mathbb{E}\left(W_{n, w}\right)+x \sqrt{\mathbb{V}\left(W_{n, w}\right)}\right)=\frac{1}{\sqrt{2 \pi}} \int_{-\infty}^{x} e^{-\frac{t^{2}}{2}} d t,
$$

where $\mathbb{E}\left(W_{n, w}\right)$ and $\mathbb{V}\left(W_{n, w}\right)$ are given in $[4]$ for $d \in\{1,2,3,4,5\}$ and in Table 1 for $d \leq 5$ and $w \leq 3$.

We remark that the main terms of the expected values for $d \in\{1,2,3\}$ are given by Avanzi [1]. In contrast to his approximate approach, our generating function approach allows us to extract lower order information as well as higher moments and to prove a central limit theorem.

\section{Simple Joint Sparse Form}

In this section we adapt the window method to exponentiation studied in [1] to the $d$-dimensional Simple Joint Sparse Form introduced in [3]. We shortly summarize the definition of this joint expansion of elements of $\mathbb{Z}^{d}$. 


\begin{tabular}{|c|cc|cc|}
\hline$(d, w)$ & $\frac{1}{n} \mathbb{E}\left(W_{n, w}\right)$ & $\frac{1}{n} \mathbb{V}\left(W_{n, w}\right)$ \\
\hline \hline$(1,1)$ & $\frac{1}{3}$ & $\approx 0.333333$ & $\frac{2}{27}$ & $\approx 0.074074$ \\
\hline$(1,2)$ & $\frac{1}{3}$ & $\approx 0.333333$ & $\frac{2}{27}$ & $\approx 0.074074$ \\
\hline$(1,3)$ & $\frac{2}{9}$ & $\approx 0.222222$ & $\frac{2}{81}$ & $\approx 0.024691$ \\
\hline$(2,1)$ & $\frac{5}{9}$ & $\approx 0.555556$ & $\frac{4}{27}$ & $\approx 0.148148$ \\
\hline$(2,2)$ & $\frac{11}{27}$ & $\approx 0.407407$ & $\frac{80}{2187}$ & $\approx 0.036580$ \\
\hline$(2,3)$ & $\frac{32}{117}$ & $\approx 0.273504$ & $\frac{2464}{177957}$ & $\approx 0.013846$ \\
\hline$(3,1)$ & $\frac{19}{27}$ & $\approx 0.703704$ & $\frac{1064}{6561}$ & $\approx 0.162170$ \\
\hline$(3,2)$ & $\frac{131}{297}$ & $\approx 0.441077$ & $\frac{196210}{8732691}$ & $\approx 0.022468$ \\
\hline$(3,3)$ & $\frac{1082}{3645}$ & $\approx 0.296845$ & $\frac{1822366}{199290375}$ & $\approx 0.009144$ \\
\hline$(4,1)$ & $\frac{65}{81}$ & $\approx 0.802469$ & $\frac{41776}{295245}$ & $\approx 0.141496$ \\
\hline$(4,2)$ & $\frac{3469}{7533}$ & $\approx 0.460507$ & $\frac{242550560}{15832158831}$ & $\approx 0.015320$ \\
\hline$(4,3)$ & $\frac{22976}{74115}$ & $\approx 0.310005$ & $\frac{95487386176}{15078376202625}$ & $\approx 0.006333$ \\
\hline$(5,1)$ & $\frac{211}{243}$ & $\approx 0.868313$ & $\frac{644320}{5845851}$ & $\approx 0.110218$ \\
\hline$(5,2)$ & $\frac{32297}{68283}$ & $\approx 0.472987$ & $\frac{7065325354906}{648539908339455}$ & $\approx 0.010894$ \\
\hline$(5,3)$ & $\frac{11961398}{37601091} \approx 0.318113$ & $\frac{963646563298519282}{218773676422818911097}$ & $\approx 0.004405$ \\
\hline
\end{tabular}

TABLE 1. Asymptotic means and variances of $W_{n, w} / n$ for small $d, w$.

In [3] it is shown that for each $d$-tuple of integers $\left(x^{(1)}, \ldots, x^{(d)}\right)$ there is a unique joint expansion $\left(\mathbf{x}_{J}, \ldots, \mathbf{x}_{2}, \mathbf{x}_{1}, \mathbf{x}_{0}\right)$, i.e.,

$$
x^{(k)}=\sum_{j=0}^{J} x_{j}^{(k)} 2^{j} \quad \text { with } \quad x_{j}^{(k)} \in\{0, \pm 1\}
$$

such that

$$
A\left(\mathbf{x}_{j+1}\right) \supsetneqq A\left(\mathbf{x}_{j}\right) \text { or } A\left(\mathbf{x}_{j+1}\right)=\emptyset, \quad 0 \leq j<J
$$

and $\mathbf{x}_{J} \neq \mathbf{0}$, where $A(\mathbf{x})$ has been defined in (2.1). This is called the Simple Joint Sparse Form of $x^{(1)}, \ldots, x^{(d)}$.

3.1. Algorithms and probabilistic model. Algorithm 3 can be used for the computation of the Simple Joint Sparse Form of $d$ integers. This algorithm was described in [3]; we need this algorithm to derive the transition probabilities for the probabilistic model we use.

From (3.1) it is clear that the Simple Joint Sparse Form can have at most $d$ consecutive nonzero digit-vectors. When applying windows to the computation of $x^{(1)} P_{1}+\cdots+x^{(d)} P_{d}$ in an Abelian group using the SJSF, it is natural to use these "guaranteed" 0 digit-vectors. Therefore we consider Algorithm 4. Its idea is as follows: we organize the Simple Joint Sparse form into blocks of non-zero columns with intermediate 0 s. These blocks can have length at most $d$ by (3.1). In order to bound the length of the look-ahead, we also allow empty blocks. Then we collect $w$ consecutive blocks to form a window, where the leftmost block has to be non-empty. Consecutive 0 s at the right end of the window are removed and treated by doublings. The remaining window is looked up in a precomputed table. Zeros between windows are treated by doublings.

Let $\mathcal{J}_{d}$ be the space of all "infinite SJSFs", i.e.,

$$
\mathcal{J}_{d}=\left\{\left(\ldots, \mathbf{x}_{1}, \mathbf{x}_{0}\right) \in\{0, \pm 1\}^{d \times \mathbb{N}_{0}} \mid \forall j \in \mathbb{N}_{0}: A\left(\mathbf{x}_{j}\right) \varsubsetneqq A\left(\mathbf{x}_{j+1}\right) \text { or } A\left(\mathbf{x}_{j+1}\right)=\emptyset\right\} .
$$

We now define a probability measure on $\mathcal{J}_{d}$ as the image of the Haar-measure on $\mathbb{Z}_{2}^{d}=\{0,1\}^{d \times \mathbb{N}_{0}}$ under the map $\mathbb{Z}_{2}^{d} \rightarrow \mathcal{J}_{d}$ given by Algorithm 3. This measure induces uniform distribution on all 

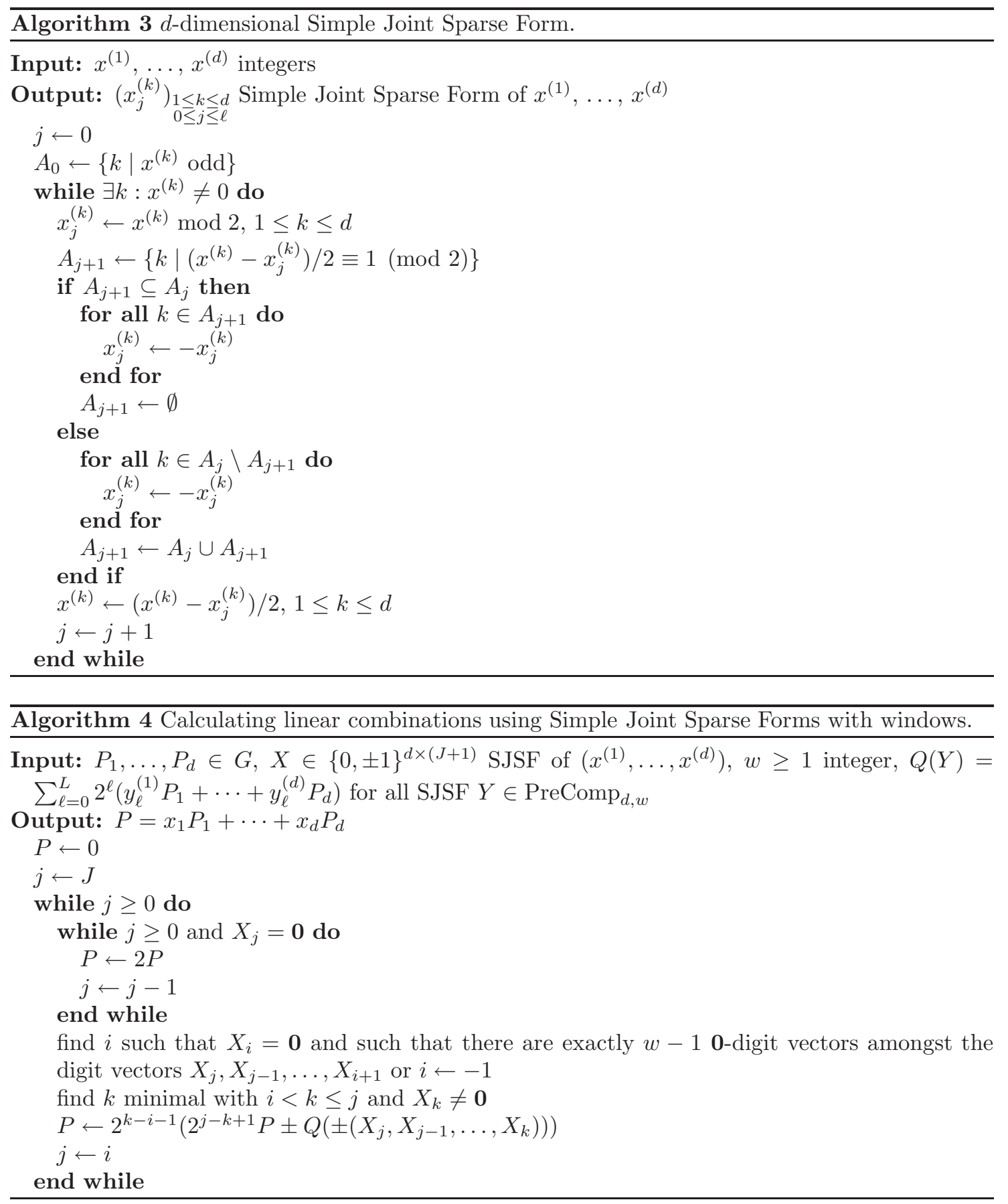

possible input vectors in $\left\{0, \ldots, 2^{N}-1\right\}^{d}$. Analyzing the congruence conditions used in Algorithm 3 yields (for $\mathbf{x}, \mathbf{y} \in\{0, \pm 1\}^{d}$ satisfying $A(\mathbf{x}) \varsubsetneqq A(\mathbf{y})$ or $\mathbf{y}=\mathbf{0}$ )

$$
\mathbb{P}\left(\mathbf{X}_{j+1}=\mathbf{y} \mid \mathbf{X}_{j}=\mathbf{x}\right)=2^{-(d+\# A(\mathbf{y})-\# A(\mathbf{x}))}
$$

and

$$
\mathbb{P}\left(\mathbf{X}_{0}=\mathbf{y}\right)=2^{-(d+\# A(\mathbf{y}))} .
$$

We are interested in the random variable $W_{n}=W_{n, w}(\mathbf{X})$, which counts the number of group additions when applying Algorithm 4 to $\left(\mathbf{X}_{n-1}, \ldots, \mathbf{X}_{0}\right)$. Since $W_{n}$ depends only on

$$
\left(\# A\left(\mathbf{X}_{n-1}\right), \ldots, \# A\left(\mathbf{X}_{0}\right)\right) \text {, }
$$


we compute the corresponding transition probabilities

$$
p_{k, \ell}:=\mathbb{P}\left(\# A\left(\mathbf{X}_{j+1}\right)=\ell \mid \# A\left(\mathbf{X}_{j}\right)=k\right)= \begin{cases}\left(\begin{array}{l}
d-k \\
\ell-k
\end{array}\right) 2^{-(d-k)} & \text { for } \ell>k, \\
2^{-(d-k)} & \text { for } \ell=0 .\end{cases}
$$

In order to study $W_{n}$ we introduce the generating function

$$
F(y, z)=\sum_{m=0}^{\infty} \sum_{n=0}^{\infty} \mathbb{P}\left(W_{n}=m\right) y^{m} z^{n} .
$$

We denote $N=\{0, \pm 1\}^{d} \backslash\{\mathbf{0}\}$. A regular expression for a window containing $w-1$ "interior $\mathbf{0}$ s" and it's delimiting right $\mathbf{0}$ is given by

$$
N\left(N^{*} \mathbf{0}\right)^{w}
$$

(we remark here that the conditions (3.1) have to be satisfied). Since adjacent windows can be separated by an arbitrary number of $\mathbf{0}$ s and windows at the end of the expansion can be incomplete, the generating function $F(y, z)$ can be calculated by

$$
\begin{aligned}
& F(y, z)=(1, \ldots, 1)\left(I-(I-V)^{-1} y U\left((I-U)^{-1} V\right)^{w}\right)^{-1}(I-V)^{-1} \\
& \quad \times\left(I+\left(y U+y U(I-U)^{-1} V+\cdots+y U\left((I-U)^{-1} V\right)^{w-1}\right)(I-U)^{-1}\right)(1,0 \ldots, 0)^{T}
\end{aligned}
$$

where

$$
\begin{aligned}
& U=z\left([k>0] p_{\ell, k}\right)_{0 \leq k, \ell \leq d}, \\
& V=z\left([k=0] p_{\ell, k}\right)_{0 \leq k, \ell \leq d} .
\end{aligned}
$$

We remark here that the "exit vector" $(1,0, \ldots, 0)$ simulates a $\mathbf{0}$ in position -1 which corresponds to the fact that $\mathbb{P}\left(\mathbf{X}_{0}=\mathbf{y}\right)$ can be computed by setting $\mathbf{x}=\mathbf{0}$ in (3.3).

For $d \leq 20$ we computed the function $F(y, z)$ using Mathematica ${ }^{\circledR}$. Only the result for $d=2$ fits on one line:

$$
\begin{aligned}
& F(y, z)= \\
& \frac{4-(4-3 y) z^{3}+z\left(4+y\left(3-2^{3-2 w}\left(z(1+z)^{2}\right)^{w}\right)\right)-z^{2}\left(4-y\left(6-4^{1-w}\left(z(1+z)^{2}\right)^{w}\right)\right)}{(1-z)\left(4-z^{3}+z\left(7-2^{3-2 w} y\left(z(1+z)^{2}\right)^{w}\right)+z^{2}\left(2-4^{1-w} y\left(z(1+z)^{2}\right)^{w}\right)\right)} .
\end{aligned}
$$

The means and variances can be computed from the generating functions as above. We did these computations for $d=1, \ldots, 20$ (cf. [4]). Table 2 gives the asymptotic main terms for $1 \leq d \leq 7$.

By the same means we compute expectation and variance of the number $W_{n, 0}$ of additions using SJSFs without windows (see Table 3).

We summarize our results in the following theorem.

Theorem 2. Let $w \geq 1, d \geq 1$ and $W_{n, w}$ be the random variable counting the number of group additions when calculating $X_{1} P_{1}+\cdots+X_{d} P_{d}$ using Algorithm 4, where $X_{1}, \ldots, X_{d}$ are independent random variables uniformly distributed on $\left\{0, \ldots, 2^{n}-1\right\}$. Then

$$
\lim _{n \rightarrow \infty} \mathbb{P}\left(W_{n, w} \leq \mathbb{E}\left(W_{n, w}\right)+x \sqrt{\mathbb{V}\left(W_{n, w}\right)}\right)=\frac{1}{\sqrt{2 \pi}} \int_{-\infty}^{x} e^{-\frac{t^{2}}{2}} d t,
$$

where $\mathbb{E}\left(W_{n, w}\right)$ and $\mathbb{V}\left(W_{n, w}\right)$ are given in [4] for $1 \leq d \leq 20$ and in Table 2 for $d \leq 7$.

3.2. Counting the precomputed values. We now count the number of elements in the set PreComp $_{d, w}$ of precomputed values. The following computations show that \# PreComp P $_{d, w}$ increases exponentially in $w$ and hyperexponentially in $d$. The set PreComp ${ }_{d, w}$ consists of all finite sequences of digit vectors satisfying (3.1), containing at most $w-1$ 0-digit vectors, and which start and end with a non-zero digit vector. Furthermore, we normalize the elements of PreComp $\operatorname{pos}_{d, w}$ by requiring that the first non-zero entry in the first column equals +1 . Since any admissible sequence of digit vectors can be followed by an arbitrary number of $\mathbf{0}$-digit vectors, we have

$$
\# \operatorname{PreComp}_{d, w}=\#\left(\left\{X \in\left(\{0, \pm 1\}^{d}\right)^{*} \mid X \in N N^{*}\left(\mathbf{0} N^{*}\right)^{w-1} \text { and } X \text { a SJSF }\right\} /\{ \pm 1\}\right) \text {, }
$$




\begin{tabular}{|l|c|c|}
\hline$d$ & $\frac{1}{n} \mathbb{E}\left(W_{n, w}\right)$ & $\frac{1}{n} \mathbb{V}\left(W_{n, w}\right)$ \\
\hline 1 & $\frac{2}{3(w+1)}$ & $\frac{2(w+7)}{27(w+1)^{3}}$ \\
\hline 2 & $\frac{3}{2(3 w+1)}$ & $\frac{9}{16(3 w+1)^{2}}$ \\
\hline 3 & $\frac{112}{39(7 w+1)}$ & $\frac{784(1225 w-137)}{59319(7 w+1)^{3}}$ \\
\hline 4 & $\frac{960}{179(15 w+1)}$ & $\frac{8640(82175 w-10751)}{5735339(15 w+1)^{3}}$ \\
\hline 5 & $\frac{63488}{6327(31 w+1)}$ & $\frac{63488(3549810031 w-337187183)}{253275687783(31 w+1)^{3}}$ \\
\hline 6 & $\frac{4128768}{218357(63 w+1)}$ & $\frac{12386304(5319844735149 w-322156222637)}{10411213601145293(63 w+1)^{3}}$ \\
\hline 7 & $\frac{1065353216}{29681427(127 w+1)}$ & $\frac{1065353216(1110439852652223895 w-40282349901979031)}{26148954556492040001483(127 w+1)^{3}}$ \\
\hline
\end{tabular}

TABLE 2. Asymptotic means and variances of $W_{n, w}$.

\begin{tabular}{|c|cc|cc|}
\hline$d$ & $\frac{1}{n} \mathbb{E}\left(W_{n, 0}\right)$ & \multicolumn{1}{|c|}{$\frac{1}{n} \mathbb{V}\left(W_{n, 0}\right)$} & \\
\hline \hline 1 & $\frac{1}{3}$ & $\approx 0.3333$ & $\frac{2}{27}$ & $\approx 0.0741$ \\
\hline 2 & $\frac{1}{2}$ & $\approx 0.5$ & $\frac{1}{16}$ & $\approx 0.0625$ \\
\hline 3 & $\frac{23}{39}$ & $\approx 0.5897$ & $\frac{2800}{59319}$ & $\approx 0.0472$ \\
\hline 4 & $\frac{115}{179}$ & $\approx 0.6425$ & $\frac{210368}{5735339}$ & $\approx 0.0367$ \\
\hline 5 & $\frac{4279}{6327}$ & $\approx 0.6763$ & $\frac{7565047808}{253275687783}$ & $\approx 0.0299$ \\
\hline 6 & $\frac{152821}{218357}$ & $\approx 0.6999$ & $\frac{263523314106368}{10411213601145293}$ & $\approx 0.0253$ \\
\hline 7 & $\frac{21292819}{29681427}$ & $\approx 0.7174$ & $\frac{577533922219434967040}{26148954556492040001483}$ & $\approx 0.0221$ \\
\hline
\end{tabular}

TABLE 3. Asymptotic means and variances of the number of additions when using SJSF without windows.

where $N=\{0, \pm 1\}^{d} \backslash\{\mathbf{0}\}$. Thus we have for $w \geq 1$

$$
\text { \# } \operatorname{PreComp}_{d, w}=\frac{1}{2}(1,0, \ldots, 0) C(I-C)^{-1}\left(B(I-C)^{-1}\right)^{w-1}(1, \ldots, 1)^{T} \text {, }
$$

where

$$
B=([\ell=0])_{0 \leq k, \ell \leq d}, \quad C=\left([\ell>k]\left(\begin{array}{c}
d-k \\
\ell-k
\end{array}\right) 2^{\ell}\right)_{0 \leq k, \ell \leq d} .
$$

In order to study the behaviour for large $d$, we study the matrix $B(I-C)^{-1}$ in more detail. It is clear that all rows of $B(I-C)^{-1}$ are equal. It can be proved by induction that the $k$-th entry in 
this row equals

$$
\left(\begin{array}{l}
d \\
k
\end{array}\right) 2^{k} a_{k}
$$

where $a_{k}$ satisfies the recursion

$$
a_{n}=\sum_{k=0}^{n-1}\left(\begin{array}{l}
n \\
k
\end{array}\right) 2^{k} a_{k} \quad n \geq 1, \quad a_{0}=1 .
$$

Since $\lambda_{d}=\sum_{k=0}^{d}\left(\begin{array}{l}d \\ k\end{array}\right) 2^{k} a_{k}=\left(2^{d}+1\right) a_{d}$ is the only non-zero eigenvalue of $B(I-C)^{-1}$, we get \# $\operatorname{PreComp}_{d, w}=\frac{1}{2}\left(\lambda_{d}-1\right) \lambda_{d}^{w-1}$.

In order to study the asymptotic behaviour of $a_{n}$ we substitute $a_{n}=n ! 2^{\left(\begin{array}{c}n \\ 2\end{array}\right)} b_{n}$. This is motivated by the fact that the dominating summand in (3.8) occurs for $k=n-1$; this gives $a_{n} \approx n 2^{n-1} a_{n-1}$. We get the recursion

$$
b_{n}=\sum_{k=0}^{n-1} \frac{1}{(n-k) !} 2^{\left(\begin{array}{c}
k+1 \\
2
\end{array}\right)-\left(\begin{array}{c}
n \\
2
\end{array}\right)} b_{k}
$$

for $b_{n}$. Since the term for $k=n-1$ on the right hand side equals $b_{n-1}$, the sequence $b_{n}$ is monotonically increasing. It remains to show that $b_{n}$ is bounded. For this purpose we estimate

$$
b_{n} \leq b_{n-1}\left(1+\sum_{k=0}^{n-2} \frac{1}{(n-k) !} 2^{\left(\begin{array}{c}
k+1 \\
2
\end{array}\right)-\left(\begin{array}{c}
n \\
2
\end{array}\right)}\right) \leq b_{n-1}\left(\sum_{k=1}^{n} \frac{1}{k !} 2^{-\frac{1}{2} n(k-1)}\right),
$$

where we have used $\left(\begin{array}{c}n \\ 2\end{array}\right)-\left(\begin{array}{c}k+1 \\ 2\end{array}\right) \geq \frac{1}{2} n(n-k-1)$. Using $\frac{e^{x}-1}{x} \leq e^{x}$ for $x>0$ and extending the finite sum on the right hand side to an infinite sum, we obtain

$$
b_{n} \leq b_{n-1} \exp \left(2^{-\frac{1}{2} n}\right) \leq b_{0} \exp \left(\sum_{k=1}^{n} 2^{-\frac{k}{2}}\right) .
$$

Thus $b_{n}$ is bounded and we can form the generating function

$$
f(z)=\sum_{n=0}^{\infty} b_{n} z^{n}
$$

Inserting the recursion (3.9) into (3.10) yields

$$
f(z)=1+\sum_{n=1}^{\infty} \sum_{k=0}^{n-1} \frac{1}{(n-k) !} 2^{\left(\begin{array}{c}
k+1 \\
2
\end{array}\right)-\left(\begin{array}{c}
n \\
2
\end{array}\right)} b_{k} z^{n}=1+\sum_{\ell=1}^{\infty} \frac{1}{\ell !} 2^{-\left(\begin{array}{c}
\ell \\
2
\end{array}\right)} z^{\ell} \sum_{k=0}^{\infty} b_{k}\left(2^{-(\ell-1)} z\right)^{k} .
$$

The inner sum in (3.11) is just $f\left(2^{-(\ell-1)} z\right)$. Furthermore, the summand for $\ell=1$ equals $z f(z)$. This yields

$$
f(z)=\frac{1}{1-z}\left(1+\sum_{\ell=1}^{\infty} \frac{1}{(\ell+1) ! 2^{\left(\begin{array}{l}
\ell+1 \\
2
\end{array}\right)}} z^{\ell+1} f\left(2^{-\ell} z\right)\right)
$$

from which we conclude that $f$ has a meromorphic continuation to the whole complex plane with simple poles at $z=2^{\ell}, \ell \in \mathbb{N}$. The residue of $f(z)$ at $z=1$ equals

$$
c=1+\sum_{\ell=1}^{\infty} \frac{1}{\left.(\ell+1) ! 2^{\left({ }_{2}+1\right.}\right)} f\left(2^{-\ell}\right)
$$

$$
=1.572986203588985421674040830458773854660492965 \ldots
$$

Putting everything together we conclude that

$$
a_{n} \sim c n ! 2^{\left(\begin{array}{c}
n \\
2
\end{array}\right) .}
$$

Summing up, we have 
Theorem 3. Let $d, w \geq 1$. Then the number of precomputed values \# PreComp ${ }_{d, w}$ needed in Algorithm 4 is given by

$$
\text { \# PreComp } \operatorname{Pow}_{d, w}=\frac{1}{2}\left(\lambda_{d}-1\right) \lambda_{d}^{w-1} \quad \text { with } \quad \lambda_{d} \sim c d ! 2^{\left(\begin{array}{c}
d+1 \\
2
\end{array}\right)} .
$$

In order to compare the number of additions when computing linear combinations using SJSF with and without windows, we introduce the notation

$$
\# \operatorname{PreComp}_{d, 0}=\#\left(\left(\{0, \pm 1\}^{d} \backslash\{\mathbf{0}\}\right) /\{ \pm 1\}\right)=\frac{3^{d}-1}{2}
$$

for the number of precomputations needed for SJSF without windows. Table 4 shows the number of precomputed values in the various situations.

\begin{tabular}{|r|l|r|}
\hline$d$ & \# PreComp $_{d, w}-d$ & \# PreComp $_{d, 0}-d$ \\
\hline 1 & $3^{w-1}-1$ & 0 \\
\hline 2 & $12 \cdot 25^{w-1}-2$ & 2 \\
\hline 3 & $301 \cdot 603^{w-1}-3$ & 10 \\
\hline 4 & $19320 \cdot 38641^{w-1}-4$ & 36 \\
\hline
\end{tabular}

TABLE 4. Number of precomputed values.

Table 5 shows the minimal values of $n$, such that

$$
\operatorname{PreComp}_{d, w}+\mathbb{E}\left(W_{n, w}\right) \leq \operatorname{PreComp}_{d, w-1}+\mathbb{E}\left(W_{n, w-1}\right) .
$$

\begin{tabular}{|r|r|r|r|}
\hline$d$ & $w=1$ & $w=2$ & $w=3$ \\
\hline 1 & 1 & 19 & 110 \\
\hline 2 & 65 & 1793 & 112001 \\
\hline 3 & 1249 & 1081666 & 1793670987 \\
\hline 4 & 62748 & 4602740129 & 511331633697389 \\
\hline
\end{tabular}

TABLE 5. Threshold numbers $n$ depending on the window size $w$ and dimension $d$.

\section{Calculating a minimal expansion from left to Right}

It is a major disadvantage of Joint Sparse Form representations of pairs of integers that they can only be computed reading the binary expansion from right to left (cf. [3, 12]). However, computing linear combinations using these representations requires the digits from left to right. Therefore, the full representation has to be precomputed and stored.

However, as in the one dimensional case (cf. $[6,8]$ ), it is possible to compute some minimal joint expansion from left to right using a transducer automaton.

We start with a special representation of the two coordinates which has the property that carries do not propagate too far. This is achieved by the condition that every two non-zero digits which are adjacent or separated by 0s only, have different sign (alternating greedy expansion). Then the expansions of the two coordinates are put together and local rules are applied to produce as many ${ }_{0}^{0}$-digits as possible. The "carry absorption property" ensures that these local changes do not affect the more significant digits. 
Definition 1. $\varepsilon \in\{-1,0,1\}^{\mathbb{N}_{0}}$ is called an alternating greedy expansion of $n \in \mathbb{Z}$, if the following conditions are satisfied:

(1) Only a finite number of $\varepsilon_{j}$ is nonzero,

(2) $n=\sum_{j \geq 0} \varepsilon_{j} 2^{j}$,

(3) If $\varepsilon_{j}=\varepsilon_{\ell} \neq 0$ for some $j<\ell$, then there is a $k$ with $j<k<\ell$ such that $\varepsilon_{j}=-\varepsilon_{k}=\varepsilon_{\ell}$.

(4) For $\underline{j}:=\min \left\{j: \varepsilon_{j} \neq 0\right\}$ and $\bar{j}:=\max \left\{j: \varepsilon_{j} \neq 0\right\}$, we have $\operatorname{sign}(n)=\varepsilon_{\bar{j}}=-\varepsilon_{\underline{j}}$.

The last condition ensures uniqueness.

Proposition 1. For any integer $n$, there is a unique alternating greedy expansion. It can be computed by Algorithm 5.

The proof is easy.

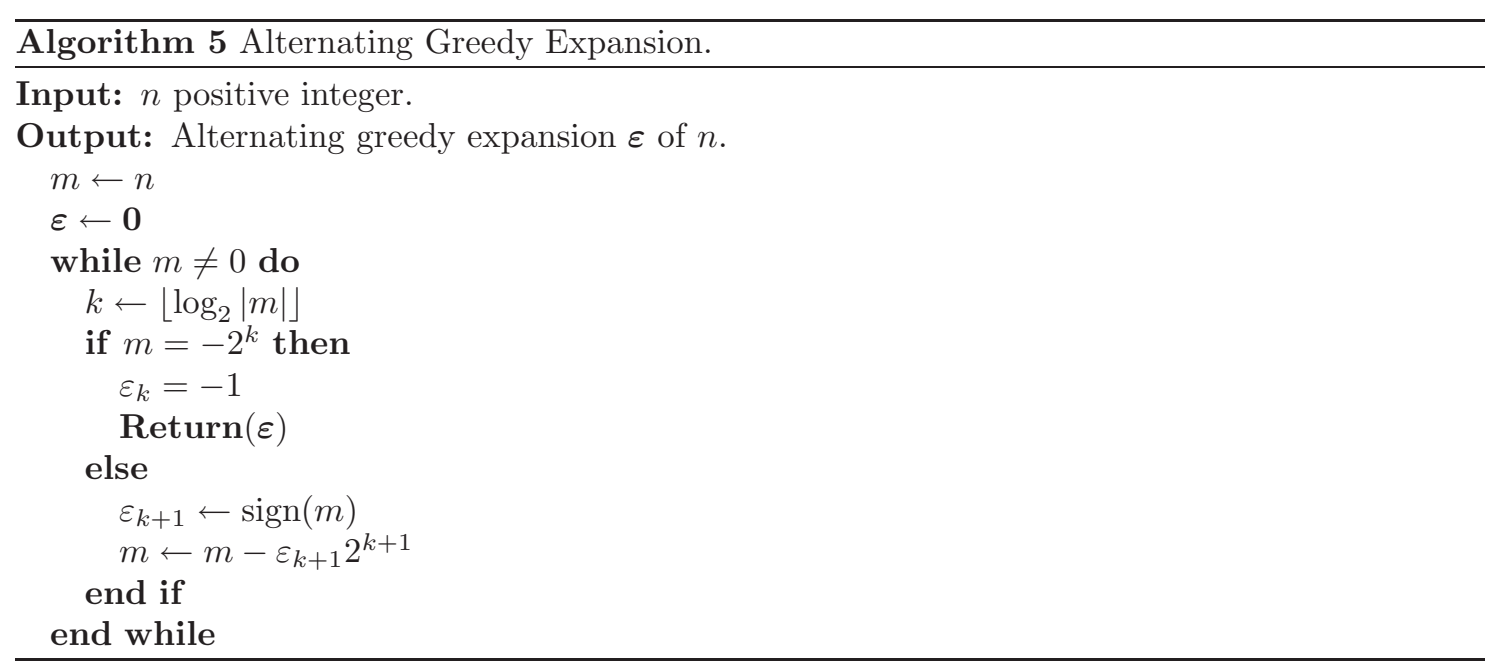

This "alternating greedy" expansion of single integers can be computed from the standard binary expansion by a transducer automaton as shown in Figure 2.

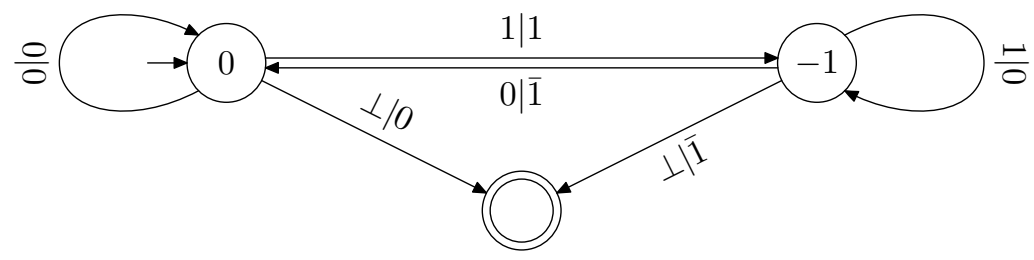

Figure 2. Transducer Automaton for computing an alternating greedy expansion from left to right. The symbol $\perp$ denotes the end of the sequence.

Now we think about a pair of integers $(x, y)$, both given in the alternating greedy expansion. When we parse this two rowed representation from left to right, we claim the following: if we

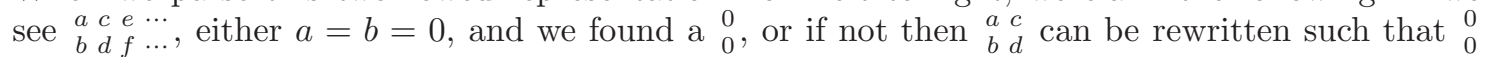
is produced, or if this is not possible, $\begin{array}{ll}a c e \\ b \quad d f\end{array}$ can be rewritten such that ${ }_{0}^{0}$ is produced. So, after at most three digits, a ${ }_{0}^{0}$ has been produced. In other words, a representation of length $J$ has the property that at least $\lfloor J / 3\rfloor$ digits are equal to ${ }_{0}^{0}$. Recall that the representation of Solinas [12] (JSF) resp. the representation in [3] (SJSF) have this property. The Table 6 contains all the necessary information. (The obvious variants when either interchanging the top resp. bottom rows or exchanging $1 \leftrightarrow \overline{1}$ are not explicitly stated.) Note that in a few instances one would have some choices. E. g., if we see $\begin{array}{lll}1 & 0 & 1 \\ 0 & 1 & 1\end{array}$, we decided to replace it by $\begin{array}{llll}1 & 0 & 1 \\ 0 & 0 & 1\end{array}$, but we could have chosen

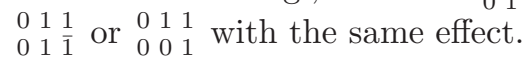




\begin{tabular}{|c|c|}
\hline Read & Write \\
\hline 0 & 0 \\
\hline 0 & 0 \\
\hline 10 & 10 \\
\hline 10 & 10 \\
\hline $1 \overline{1}$ & 01 \\
\hline $1 \overline{1}$ & 01 \\
\hline $1 \overline{1} 0$ & $1 \overline{1} 0$ \\
\hline 100 & 100 \\
\hline $1 \overline{1} 0$ & 010 \\
\hline $10 \overline{1}$ & 011 \\
\hline $1 \overline{1} 1$ & $10 \overline{1}$ \\
\hline 100 & 100 \\
\hline $1 \overline{1} 1$ & 011 \\
\hline $10 \overline{1}$ & $\begin{array}{lll}0 & 1 & 1\end{array}$ \\
\hline $1 \overline{1}$ & 01 \\
\hline $0 x$ & $0 x$ \\
\hline 100 & 100 \\
\hline 010 & 010 \\
\hline 100 & 100 \\
\hline $01 \overline{1}$ & $\begin{array}{lll}0 & 0 & 1\end{array}$ \\
\hline $10 \overline{1}$ & 011 \\
\hline $\begin{array}{lll}0 & 10\end{array}$ & 010 \\
\hline $10 \overline{1}$ & $10 \overline{1}$ \\
\hline $01 \overline{1}$ & $\begin{array}{lll}0 & 0 & 1\end{array}$ \\
\hline
\end{tabular}

TABLE 6. Rules for modifying a pair of alternating greedy expansions to a minimal joint expansion.

Clearly, this can be realized by a transducer automaton, too.

Combining the two transducer automata, we get a single transducer automaton to calculate a low weight expansion from the binary expansions of two integers. The resulting transducer automaton is shown in Table 7 and in Figure 3 (note that the final state has not been drawn in Figure 3).

We now prove that this transducer indeed calculates a minimal joint expansion. To this aim, we denote by $h(x, y)$ and $\widetilde{h}(x, y)$ the joint Hamming weights of the SJSF and the output of the transducer for $x$ and $y$, respectively. We set

$$
\begin{aligned}
w_{k n} & :=\#\left\{(x, y) \mid 0 \leq x, y<2^{n}, h(x, y)=k\right\}, \\
\widetilde{w}_{k n} & :=\#\left\{(x, y) \mid 0 \leq x, y<2^{n}, \widetilde{h}(x, y)=k\right\}, \\
F(Y, Z) & :=\sum_{k, n \geq 0} w_{k n} Y^{k} Z^{n}, \\
\widetilde{F}(Y, Z) & :=\sum_{k, n \geq 0} \widetilde{w}_{k n} Y^{k} Z^{n} .
\end{aligned}
$$

Let $\widetilde{m}_{i j}^{(\delta \varepsilon)}:=Y^{h}$, where $h$ is the joint Hamming weight of the transition $j=\left(\begin{array}{l}\delta \\ \varepsilon\end{array}\right) . i$ in the transducer. If there is no such transition, we set $\widetilde{m}_{i j}^{(\delta \varepsilon)}:=0$. We set $\widetilde{M}^{(\delta, \varepsilon)}:=\left(\widetilde{m}_{i j}^{(\delta \varepsilon)}\right)_{1 \leq i, j \leq 32}$ and $\widetilde{M}:=$ $\sum_{0 \leq \delta, \varepsilon \leq 1} \widetilde{M}^{(\delta, \varepsilon)}$. Then it is easily seen that

$$
\widetilde{F}(Y, Z)=(1,0, \ldots, 0) \cdot(I-Z \widetilde{M})^{-1} \cdot\left(\widetilde{M}^{(00)}\right)^{2} \cdot(1,0, \ldots, 0)^{T} .
$$

The factors $\widetilde{M}^{(00)}$ ensure that we return to state 1 writing all accumulated digits. An explicit calculation yields

$$
\widetilde{F}(Y, Z)=\frac{-1+(2-3 Y) Z+\left(-1+13 Y-9 Y^{2}\right) Z^{2}+Y\left(-10+37 Y-18 Y^{2}\right) Z^{3}-2 Y^{2}\left(16-23 Y+8 Y^{2}\right) Z^{4}+8 Y^{3}(-4+3 Y) Z^{5}}{(-1+Z)\left(-1+Z+2 Y Z^{2}\right)\left(-1+Z+8 Y Z^{2}+16 Y^{2} Z^{3}\right)} .
$$

A similar calculation using the right-to-left transducer described in [3] yields

$$
\widetilde{F}(Y, Z)=F(Y, Z) \text {. }
$$




\begin{tabular}{|c|c|c|c|c|c|c|c|c|c|c|c|}
\hline & & & & In: & & In: & & & & & \\
\hline State & $\mathrm{Nr}$ & Out: & To: & Out: & To: & Out: & To: & Out: & To: & Out: & To: \\
\hline $\begin{array}{l}0 \\
0\end{array}$ & 1 & $\begin{array}{l}0 \\
0\end{array}$ & 1 & $\varepsilon$ & 3 & $\varepsilon$ & 4 & $\varepsilon$ & 5 & $\begin{array}{l}0 \\
0\end{array}$ & 2 \\
\hline$\perp$ & 2 & $\varepsilon$ & 2 & $\varepsilon$ & 2 & $\varepsilon$ & 2 & $\varepsilon$ & 2 & $\varepsilon$ & 2 \\
\hline $\begin{array}{ll}1 & \overline{1} \\
0 & 0\end{array}$ & 3 & $\begin{array}{lll}0 & 1 \\
0 & 0\end{array}$ & 1 & $\begin{array}{ll}1 & 0 \\
0 & 0\end{array}$ & 6 & $\begin{array}{ll}0 & 1 \\
0 & 1\end{array}$ & 7 & $\varepsilon$ & 8 & $\begin{array}{ll}0 & 1 \\
0 & 0\end{array}$ & 2 \\
\hline $\begin{array}{ll}0 & 0 \\
1 & \overline{1}\end{array}$ & 4 & $\begin{array}{lll}0 & 0 \\
0 & 1\end{array}$ & 1 & $\begin{array}{ll}0 & 1 \\
0 & 1\end{array}$ & 6 & $\begin{array}{ll}0 & 0 \\
1 & 0\end{array}$ & 7 & $\varepsilon$ & 9 & $\begin{array}{ll}0 & 0 \\
0 & 1\end{array}$ & 2 \\
\hline $\begin{array}{ll}1 & \overline{1} \\
1 & \overline{1}\end{array}$ & 5 & $\begin{array}{ll}0 & 1 \\
0 & 1\end{array}$ & 1 & $\varepsilon$ & 10 & $\varepsilon$ & 11 & $\begin{array}{ll}1 & 0 \\
1 & 0\end{array}$ & 12 & $\begin{array}{ll}0 & 1 \\
0 & 1\end{array}$ & 2 \\
\hline $\begin{array}{l}\overline{1} \\
0\end{array}$ & 6 & $\varepsilon$ & 13 & $\begin{array}{l}0 \\
0\end{array}$ & 6 & $\varepsilon$ & 14 & $\varepsilon$ & 15 & $\begin{array}{l}\overline{1} \\
0\end{array}$ & 2 \\
\hline$\frac{0}{1}$ & 7 & $\varepsilon$ & 16 & $\varepsilon$ & 17 & $\begin{array}{l}0 \\
0\end{array}$ & 7 & $\varepsilon$ & 18 & $\frac{0}{1}$ & 2 \\
\hline $\begin{array}{lll}1 & 0 & \overline{1} \\
0 & 1 & \overline{1}\end{array}$ & 8 & $\begin{array}{lll}1 & 0 & \overline{1} \\
0 & 0 & 1\end{array}$ & 1 & $\begin{array}{lll}1 & 0 & 0 \\
0 & 0 & 1\end{array}$ & 6 & $\begin{array}{lll}0 & 1 & 1 \\
0 & 1 & 0\end{array}$ & 7 & $\begin{array}{lll}1 & 0 & 0 \\
0 & 1 & 0\end{array}$ & 12 & $\begin{array}{lll}1 & 0 & \overline{1} \\
0 & 0 & 1\end{array}$ & 2 \\
\hline $\begin{array}{lll}0 & 1 & \overline{1} \\
1 & 0 & \overline{1}\end{array}$ & 9 & $\begin{array}{lll}0 & 0 & 1 \\
1 & 0 & 1\end{array}$ & 1 & $\begin{array}{lll}0 & 1 & 0 \\
0 & 1 & 1\end{array}$ & 6 & $\begin{array}{lll}0 & 0 & 1 \\
1 & 0 & 0\end{array}$ & 7 & $\begin{array}{lll}0 & 1 & 0 \\
1 & 0 & 0\end{array}$ & 12 & $\begin{array}{lll}0 & 0 & 1 \\
1 & 0 & \overline{1}\end{array}$ & 2 \\
\hline $\begin{array}{lll}1 & 0 & \overline{1} \\
1 & \overline{1} & 0\end{array}$ & 10 & $\begin{array}{lll}0 & 1 & 1 \\
0 & 1 & 0\end{array}$ & 1 & $\begin{array}{lll}1 & 0 & 0 \\
1 & \overline{1} & 0\end{array}$ & 6 & $\begin{array}{lll}0 & 1 & 1 \\
0 & 1 & 1\end{array}$ & 7 & $\begin{array}{lll}1 & 0 & 0 \\
1 & 0 & 1\end{array}$ & 12 & $\begin{array}{lll}0 & 1 & 1 \\
0 & 1 & 0\end{array}$ & 2 \\
\hline $\begin{array}{lll}1 & \overline{1} & 0 \\
1 & 0 & \overline{1}\end{array}$ & 11 & $\begin{array}{lll}0 & 1 & 0 \\
0 & 1 & 1\end{array}$ & 1 & $\begin{array}{lll}0 & 1 & 1 \\
0 & 1 & 1\end{array}$ & 6 & $\begin{array}{lll}1 & \overline{1} & 0 \\
1 & 0 & 0\end{array}$ & 7 & $\begin{array}{lll}1 & 0 & \overline{1} \\
1 & 0 & 0\end{array}$ & 12 & $\begin{array}{lll}0 & 1 & 0 \\
0 & 1 & 1\end{array}$ & 2 \\
\hline$\overline{1}$ & 12 & $\varepsilon$ & 19 & $\varepsilon$ & 20 & $\varepsilon$ & 21 & $\begin{array}{l}0 \\
0\end{array}$ & 12 & $\overline{\overline{1}}$ & 2 \\
\hline $\begin{array}{ll}\overline{1} & 0 \\
0 & 0\end{array}$ & 13 & $\begin{array}{ll}\overline{1} & 0 \\
0 & 0\end{array}$ & 1 & $\begin{array}{ll}0 & \overline{1} \\
0 & 0\end{array}$ & 6 & $\varepsilon$ & 22 & $\begin{array}{ll}0 & \overline{1} \\
0 & 1\end{array}$ & 12 & $\begin{array}{ll}\overline{1} & 0 \\
0 & 0\end{array}$ & 2 \\
\hline $\begin{array}{ll}\overline{1} & 0 \\
1 & \overline{1}\end{array}$ & 14 & $\varepsilon$ & 23 & $\begin{array}{ll}0 & \overline{1} \\
0 & 1\end{array}$ & 6 & $\begin{array}{ll}\overline{1} & 0 \\
1 & 0\end{array}$ & 7 & $\varepsilon$ & 24 & $\begin{array}{ll}\overline{1} & 0 \\
1 & \overline{1}\end{array}$ & 2 \\
\hline $\begin{array}{ll}0 & \overline{1} \\
1 & \overline{1}\end{array}$ & 15 & $\begin{array}{ll}0 & \overline{1} \\
0 & 1\end{array}$ & 1 & $\begin{array}{ll}0 & 0 \\
0 & 1\end{array}$ & 6 & $\varepsilon$ & 25 & $\begin{array}{ll}0 & 0 \\
1 & 0\end{array}$ & 12 & $\begin{array}{ll}0 & \overline{1} \\
0 & 1\end{array}$ & 2 \\
\hline $\begin{array}{ll}0 & 0 \\
\overline{1} & 0\end{array}$ & 16 & $\begin{array}{lll}0 & 0 \\
\overline{1} & 0\end{array}$ & 1 & $\varepsilon$ & 26 & $\begin{array}{ll}0 & 0 \\
0 & \overline{1}\end{array}$ & 7 & $\begin{array}{ll}0 & \frac{1}{1} \\
0 & \overline{1}\end{array}$ & 12 & $\begin{array}{ll}0 & 0 \\
1 & 0\end{array}$ & 2 \\
\hline $\begin{array}{l}1 \overline{1} \\
\overline{1} 0\end{array}$ & 17 & $\varepsilon$ & 27 & $\begin{array}{l}10 \\
\overline{1}\end{array}$ & 6 & $\begin{array}{ll}0 & \frac{1}{1} \\
0 & \overline{1}\end{array}$ & 7 & $\varepsilon$ & 28 & $\begin{array}{l}1 \overline{1} \\
\overline{1} \\
0\end{array}$ & 2 \\
\hline $\begin{array}{l}1 \overline{1} \\
0 \overline{1}\end{array}$ & 18 & $\begin{array}{ll}0 & \frac{1}{1} \\
0 & \overline{1}\end{array}$ & 1 & $\varepsilon$ & 29 & $\begin{array}{lll}0 & 1 \\
0 & 0\end{array}$ & 7 & $\begin{array}{ll}1 & 0 \\
0 & 0\end{array}$ & 12 & $\begin{array}{ll}0 & 1 \\
0 & \frac{1}{1}\end{array}$ & 2 \\
\hline $\begin{array}{ll}\overline{1} & 0 \\
\overline{1} & 0\end{array}$ & 19 & $\begin{array}{ll}\overline{1} & 0 \\
\overline{1} & 0\end{array}$ & 1 & $\varepsilon$ & 30 & $\varepsilon$ & 31 & $\begin{array}{ll}0 & \overline{1} \\
0 & \overline{1}\end{array}$ & 12 & $\begin{array}{ll}\overline{1} & 0 \\
\overline{1} & 0\end{array}$ & 2 \\
\hline $\begin{array}{ll}0 & \overline{1} \\
\overline{1} & 0\end{array}$ & 20 & $\varepsilon$ & 32 & $\begin{array}{ll}0 & 0 \\
1 & 0\end{array}$ & 6 & $\begin{array}{ll}0 & \overline{1} \\
0 & \overline{1}\end{array}$ & 7 & $\begin{array}{ll}0 & 0 \\
0 & \overline{1}\end{array}$ & 12 & $\begin{array}{ll}0 & \overline{1} \\
\overline{1} & 0\end{array}$ & 2 \\
\hline $\begin{array}{ll}\overline{1} & 0 \\
0 & \overline{1}\end{array}$ & 21 & $\varepsilon$ & 33 & $\begin{array}{l}0 \overline{1} \\
0 \overline{1}\end{array}$ & 6 & $\begin{array}{ll}\overline{1} & 0 \\
0 & 0\end{array}$ & 7 & $\begin{array}{ll}0 & \overline{1} \\
0 & 0\end{array}$ & 12 & $\begin{array}{ll}\overline{1} & 0 \\
0 & \overline{1}\end{array}$ & 2 \\
\hline $\begin{array}{lll}\overline{1} & 0 & 0 \\
0 & 1 & \overline{1}\end{array}$ & 22 & $\begin{array}{lll}\overline{1} & 0 & 0 \\
0 & 0 & 1\end{array}$ & 1 & $\begin{array}{lll}\overline{1} & 0 & 1 \\
0 & 0 & 1\end{array}$ & 6 & $\begin{array}{lll}\overline{1} & 0 & 0 \\
0 & 1 & 0\end{array}$ & 7 & $\begin{array}{llll}0 & \overline{1} & \overline{1} \\
0 & 1 & 0\end{array}$ & 12 & $\begin{array}{lll}\overline{1} & 0 & 0 \\
0 & 0 & 1\end{array}$ & 2 \\
\hline $\begin{array}{lll}\overline{1} & 0 & 0 \\
1 & \overline{1} & 0\end{array}$ & 23 & $\begin{array}{lll}\overline{1} & 0 & 0 \\
1 & \overline{1} & 0\end{array}$ & 1 & $\begin{array}{llll}0 & \overline{1} & \overline{1} \\
0 & 1 & 0\end{array}$ & 6 & $\begin{array}{lll}\overline{1} & 0 & 0 \\
1 & 0 & \overline{1}\end{array}$ & 7 & $\begin{array}{llll}0 & \overline{1} & \overline{1} \\
0 & 1 & 1\end{array}$ & 12 & $\begin{array}{lll}\overline{1} & 0 & 0 \\
1 & \overline{1} & 0\end{array}$ & 2 \\
\hline $\begin{array}{lll}\overline{1} & 1 & \overline{1} \\
1 & 0 & \overline{1}\end{array}$ & 24 & $\begin{array}{lll}0 & \overline{1} & \overline{1} \\
0 & 1 & 1\end{array}$ & 1 & $\begin{array}{lll}0 & \overline{1} & 0 \\
0 & 1 & 1\end{array}$ & 6 & $\begin{array}{lll}\overline{1} & 0 & 1 \\
1 & 0 & 0\end{array}$ & 7 & $\begin{array}{lll}\overline{1} & 1 & 0 \\
1 & 0 & 0\end{array}$ & 12 & $\begin{array}{lll}0 & \overline{1} & \overline{1} \\
0 & 1 & 1\end{array}$ & 2 \\
\hline $\begin{array}{llll}0 & \overline{1} & 0 \\
1 & 0 & 1\end{array}$ & 25 & $\begin{array}{lll}0 & \overline{1} & 0\end{array}$ & 1 & $\begin{array}{lll}0 & 0 & \overline{1} \\
\end{array}$ & 6 & $\begin{array}{lll}0 & \overline{1} 0\end{array}$ & 7 & $\begin{array}{llll}0 & 0 & \overline{1}\end{array}$ & 12 & $\begin{array}{lll}0 & \overline{1} & 0\end{array}$ & 2 \\
\hline $\begin{array}{lll}1 & 0 & \overline{1} \\
0 & 1 & \overline{1}\end{array}$ & 26 & $\begin{array}{llll}0 & 1 & 1 \\
0 & 0 & 1\end{array}$ & 1 & $\begin{array}{lll}1 & 0 & 1 \\
0 & 1 & 0\end{array}$ & & $\begin{array}{lll}1 & 0 & 0 \\
0 & 0 & 1\end{array}$ & & $\begin{array}{lll}1 & 1 & 0\end{array}$ & & $\begin{array}{lll}0 & 1 & 1 \\
0 & 0 & 1\end{array}$ & 2 \\
\hline $\begin{array}{llll}\overline{1} & 0 & 0\end{array}$ & 26 & $\begin{array}{lllll}\overline{1} & 0 & 0\end{array}$ & 1 & $\begin{array}{lll}\overline{1} & 0 & 0 \\
& \end{array}$ & 6 & $\overline{1} 01$ & 7 & $0 \overline{1} \overline{1}$ & 12 & $\begin{array}{lll}\overline{1} & 0 & 0 \\
1 & \overline{1} & 0\end{array}$ & \\
\hline $\begin{array}{lll}1 & \overline{1} & 0 \\
\overline{1} & 0 & 0\end{array}$ & 27 & $\begin{array}{lll}1 & \overline{1} & 0 \\
\overline{1} & 0 & 0\end{array}$ & 1 & $\begin{array}{llll}1 & 0 & \overline{1} \\
\overline{1} & 0 & 0\end{array}$ & 6 & $\begin{array}{lll}0 & 1 & 0 \\
0 & \overline{1} & \overline{1}\end{array}$ & 7 & $\begin{array}{llll}0 & 1 & 1 \\
0 & \overline{1} & \frac{1}{1}\end{array}$ & 12 & $\begin{array}{lll}1 & \overline{1} & 0 \\
\overline{1} & 0 & 0\end{array}$ & 2 \\
\hline $\begin{array}{lll}1 & 0 & \overline{1} \\
\overline{1} & 1 & \overline{1}\end{array}$ & 28 & $\begin{array}{lll}0 & 1 & 1 \\
0 & \overline{1} & \overline{1}\end{array}$ & 1 & $\begin{array}{lllll}1 & 0 & 0 \\
1 & 0 & 1\end{array}$ & 6 & $\begin{array}{lll}0 & 1 & 1 \\
0 & \overline{1} & 0\end{array}$ & 7 & $\begin{array}{lll}1 & 0 & 0 \\
1 & 1 & 0\end{array}$ & 12 & $\begin{array}{lll}0 & 1 & 1 \\
0 & \overline{1} & \overline{1}\end{array}$ & 2 \\
\hline $\begin{array}{lll}1 & 0 & \overline{1} \\
0 & \overline{1} & 0\end{array}$ & 29 & $\begin{array}{lll}0 & 1 & 1 \\
0 & \overline{1} & 0\end{array}$ & 1 & $\begin{array}{lll}1 & 0 & 0 \\
0 & \overline{1} & 0\end{array}$ & 6 & $\begin{array}{lll}1 & 0 & \overline{1} \\
0 & 0 & \overline{1}\end{array}$ & 7 & $\begin{array}{lll}1 & 0 & 0 \\
0 & 0 & \overline{1}\end{array}$ & 12 & $\begin{array}{lll}0 & 1 & 1 \\
0 & \overline{1} & 0\end{array}$ & 2 \\
\hline $\begin{array}{llll}\overline{1} & 1 & \overline{1} \\
\overline{1} & 0 & 0\end{array}$ & 30 & $\begin{array}{lll}\overline{1} & 0 & 1 \\
\overline{1} & 0 & 0\end{array}$ & 1 & $\begin{array}{llll}\overline{1} & 1 & 0 \\
\overline{1} & 0 & 0\end{array}$ & 6 & $\begin{array}{lll}0 & \overline{1} & \overline{1} \\
0 & \overline{1} & \overline{1}\end{array}$ & 7 & $\begin{array}{lll}0 & \overline{1} & 0 \\
0 & \overline{1} & \overline{1}\end{array}$ & 12 & $\begin{array}{lll}\overline{1} & 0 & 1 \\
\overline{1} & 0 & 0\end{array}$ & 2 \\
\hline $\begin{array}{lll}\overline{1} & 0 & 0 \\
\overline{1} & 1 & \overline{1}\end{array}$ & 31 & $\begin{array}{lll}\overline{1} & 0 & 0 \\
\overline{1} & 0 & 1\end{array}$ & 1 & $\begin{array}{ll}0 & \overline{1} \\
0 & \overline{1} \\
\overline{1} & \overline{1}\end{array}$ & 6 & $\begin{array}{lll}\overline{1} & 0 & 0 \\
\overline{1} & 1 & 0\end{array}$ & 7 & $\begin{array}{lll}0 & \overline{1} & \overline{1} \\
0 & \overline{1} & 0\end{array}$ & 12 & $\begin{array}{llll}\overline{1} & 0 & 0 \\
\overline{1} & 0 & 1\end{array}$ & 2 \\
\hline $\begin{array}{lll}0 & \overline{1} & 0 \\
\overline{1} & 0 & 0\end{array}$ & 32 & $\begin{array}{lll}0 & \overline{1} & 0 \\
\overline{1} & 0 & 0\end{array}$ & 1 & $\begin{array}{lll}0 & 0 & \overline{1} \\
\overline{1} & 0 & 0\end{array}$ & 6 & $\begin{array}{lll}0 & \overline{1} & 0 \\
0 & \overline{1} & \overline{1}\end{array}$ & 7 & $\begin{array}{lll}0 & 0 & \overline{1} \\
\overline{1} & 0 & 1\end{array}$ & 12 & $\begin{array}{lll}0 & \overline{1} & 0 \\
\overline{1} & 0 & 0\end{array}$ & 2 \\
\hline $\begin{array}{lll}1 & 0 & 0 \\
1 & 0 & 0\end{array}$ & & $\begin{array}{lll}1 & 0 & 0 \\
1 & 0 & 0\end{array}$ & 1 & $0 \overline{1} \overline{1}$ & 6 & $\begin{array}{lll}\overline{1} & 1 & 1 \\
1 & 0\end{array}$ & 7 & $\begin{array}{lll}1 & 0 & 1 \\
1 & 0 & 1\end{array}$ & 12 & $\begin{array}{lll}1 & 0 & 0 \\
1 & 0 & 0\end{array}$ & $?$ \\
\hline $0 \overline{1} 0$ & 33 & $0 \overline{1} 0$ & 1 & $0 \overline{1} 0$ & 6 & $\begin{array}{lll}0 & 0 \overline{1}\end{array}$ & 7 & $00 \overline{1}$ & 12 & $0 \overline{1} 0$ & 2 \\
\hline
\end{tabular}

TABLE 7. Transducer automaton for calculating a minimal joint expansion from the binary expansion from left to right. The symbol $\perp$ denotes the end of the sequence. 


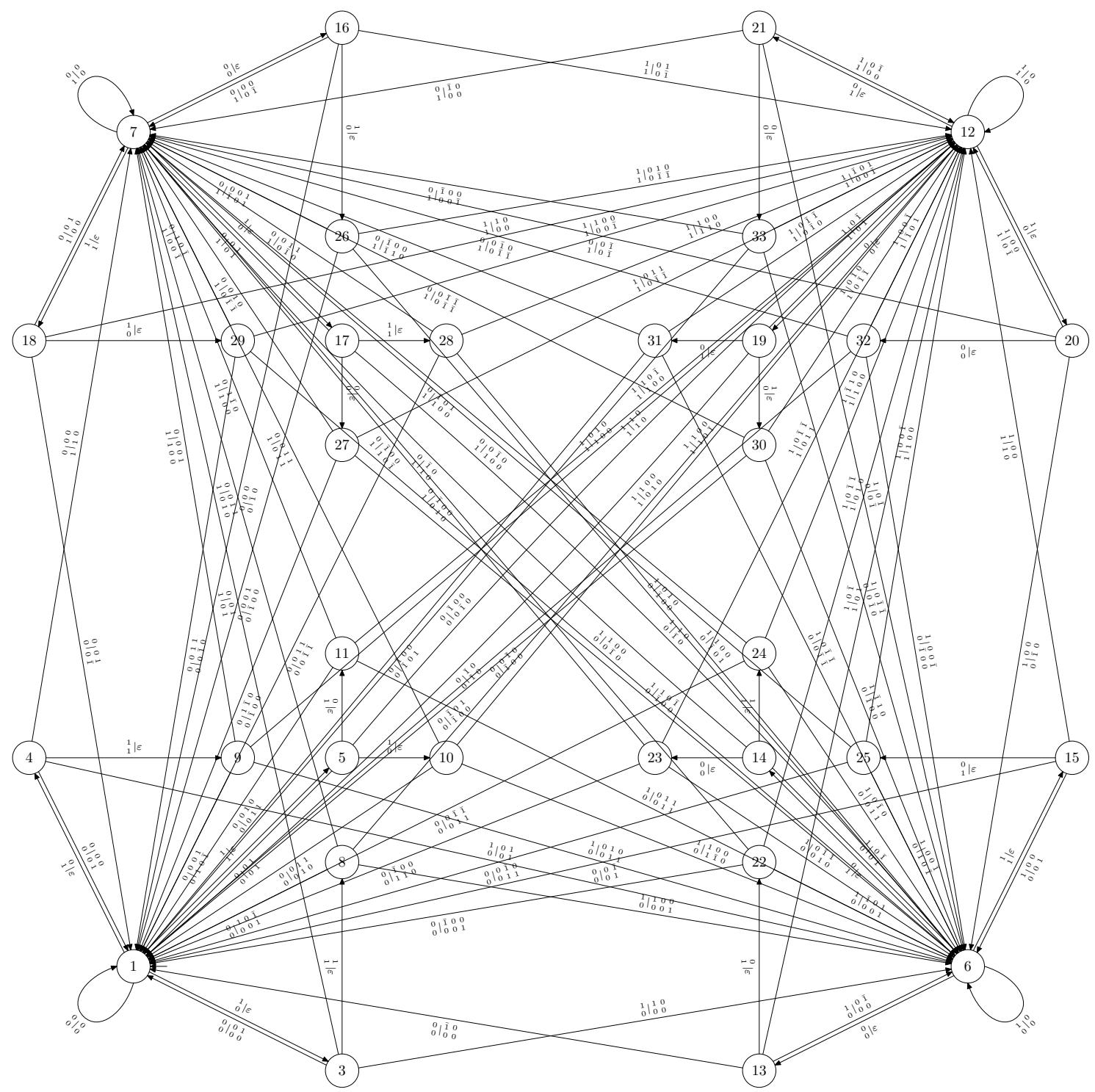

FiguRE 3. Transducer automaton for calculating a minimal joint expansion from the binary expansion.

This implies $\widetilde{w}_{k n}=w_{k n}$ for all nonnegative $k$ and $n$. Since $\widetilde{h}(x, y) \geq h(x, y)$ for all $x, y$, this proves that $\widetilde{h}(x, y)=h(x, y)$, as required.

So we proved the following theorem.

Theorem 4. Let $x, y \in \mathbb{Z}$ with binary expansions $x=\sum_{j=0}^{J} x_{j} 2^{j}$ and $y=\sum_{j=0}^{J} y_{j} 2^{j}$. Then the output $\left(\varepsilon_{J+1} \ldots \varepsilon_{0}\right)$ of the transducer in Table 7 when reading $\left({ }_{y_{J}}^{x_{J}} \ldots{ }_{y_{0}}^{x_{0}} \perp\right)$ is a joint expansion of $x$ and $y$ of minimal joint Hamming weight.

\section{REFERENCES}

[1] R. M. Avanzi, On the complexity of certain multi-exponentiation techniques in cryptography, To appear in Journal of Cryptology. Preprint available at http://www.arehcc.com/download/expo.pdf.

[2] D. M. Gordon, A survey of fast exponentiation methods, J. Algorithms 27 (1998), no. 1, 129-146.

[3] P. J. Grabner, C. Heuberger, and H. Prodinger, Distribution results for low-weight binary representations for pairs of integers, Theor. Comput. Sci. 319 (2004), 307-331. 
[4] P. J. Grabner, C. Heuberger, H. Prodinger, and J. Thuswaldner, Analysis of linear combination algorithms in cryptography - online resources, http://www.opt.math.tu-graz.ac.at/ cheub/publications/ECLinComb/.

[5] R. L. Graham, D. E. Knuth, and O. Patashnik, Concrete mathematics. A foundation for computer science, second ed., Addison-Wesley, 1994.

[6] C. Heuberger, Minimal expansions in redundant number systems: Fibonacci bases and greedy algorithms, Period. Math. Hungar. 49 (2004), 65-89.

[7] H.-K. Hwang, On convergence rates in the central limit theorems for combinatorial structures, European J. Combin. 19 (1998), 329-343.

[8] M. Joye and S.-M. Yen, Optimal left-to-right binary signed digit recoding, IEEE Transactions on Computers 49 (2000), 740-748.

[9] N. Koblitz, A. Menezes, and S. Vanstone, The state of elliptic curve cryptography, Des. Codes Cryptogr. 19 (2000), 173-193.

[10] F. Morain and J. Olivos, Speeding up the computations on an elliptic curve using addition-subtraction chains, RAIRO Inform. Théor. Appl. 24 (1990), 531-543.

[11] G. W. Reitwiesner, Binary arithmetic, Advances in computers, Vol. 1, Academic Press, New York, 1960, pp. $231-308$

[12] J. A. Solinas, Low-weight binary representations for pairs of integers, Tech. Report CORR 2001-41, University of Waterloo, 2001, available at http://www.cacr.math.uwaterloo.ca/techreports/2001/corr2001-41.ps.

[13] J. M. Thuswaldner, Summatory functions of digital sums occurring in cryptography, Period. Math. Hungar. 38 (1999), 111-130.

[14] J. von zur Gathen and J. Gerhard, Modern computer algebra, Cambridge University Press, New York, 1999.

(P. Grabner) Institut für Mathematik A, Technische Universität Graz, Steyrergasse 30,8010 Graz, Austria

E-mail address: peter.grabner@tugraz.at

(C. Heuberger) Institut für Mathematik B, Technische Universität Graz, Steyrergasse 30,8010 Graz, Austria

E-mail address: clemens.heuberger@tugraz.at

(H. Prodinger) Centre for Applicable Analysis and Number Theory, School of Mathematics, UniverSity of the Witwatersrand, P. O. Wits, 2050 Johannesburg, South Africa

E-mail address: hproding@sun.ac.za

(J. Thuswaldner) Institut für Mathematik und Angewandte Geometrie, Montanuniversität Leoben, Franz-Josef-Strasse 18, 8700 Leoben, Austria

E-mail address: Joerg. Thuswaldner@unileoben.ac.at 